\title{
Failure to confirm association between $P D C D 1$ polymorphisms and rheumatoid arthritis in a Japanese population
}

\author{
Takuji Iwamoto $\cdot$ Katsunori Ikari $\cdot$ Eisuke Inoue $\cdot$ \\ Yoshiaki Toyama · Masako Hara $\cdot$ Hisashi Yamanaka • \\ Taisuke Tomatsu $\cdot$ Shigeki Momohara $\cdot$ Naoyuki Kamatani
}

Received: 9 February 2007 / Accepted: 14 March 2007/Published online: 28 April 2007

(C) The Japan Society of Human Genetics and Springer 2007

\begin{abstract}
Programmed cell death 1 (PDCD1) is a necessary negative regulator to maintain peripheral tolerance and is a key molecule in the development of autoimmune diseases. Although PDCDI gene polymorphisms and haplotypes were reported to be associated with rheumatoid arthritis (RA), replication studies later on showed conflicting results. Here, we analyzed the association of PDCD1 with RA using a large series of Japanese RA patients and population-based controls. DNA samples were obtained from 1,504 RA patients and 449 sex-matched controls. All samples were genotyped for three SNPs on PDCD1 (PD-1.1, PD-1.3 and PD-1.5) using the TaqMan fluorogenic $5^{\prime}$ nuclease assay. Chi-square testing was performed for a case-control study, and the PENHAPLO program was used for haplotype estimation. We could not observe any significant association of PD-1.1 or PD-1.5 polymorphisms between RA. PD-1.3, which was reported to be involved in susceptibility to RA in patients of European descent, was non-polymorphic in the Japanese population. We conclude that polymorphisms in the $P D C D 1$ gene analyzed here are not associated with RA in a Japanese population.
\end{abstract}

Keywords Rheumatoid arthritis - PDCD1 - Gene · IORRA

T. Iwamoto $\cdot$ K. Ikari $(\bowtie) \cdot$ E. Inoue $\cdot$ M. Hara

H. Yamanaka $\cdot$ T. Tomatsu $\cdot$ S. Momohara .

N. Kamatani

Institute of Rheumatology, Tokyo Women's Medical University,

10-22 Kawada, Shinjuku, Tokyo 162-0054, Japan

e-mail: kikari@ior.twmu.ac.jp

T. Iwamoto · Y. Toyama

Department of Orthopedic Surgery, School of Medicine,

Keio University, Tokyo, Japan

\section{Introduction}

Rheumatoid arthritis [RA (MIM 180300)] is a chronic inflammatory autoimmune disease characterized by pathological infiltration of lymphocytes in target organs. Although the pathogenesis of RA remains unclear, it is considered that dysregulated lymphocyte activation is implicated in their pathogenesis via the breakdown of self tolerance, and multiple genetic and environmental factors are important in the development of RA.

Programmed cell death 1 (PDCD1) is a member of the CD28 family receptors that contains an immunoreceptor tyrosine-based inhibitory motif in its cytoplasmic tail and is induced on the surface of activated $\mathrm{T}$ cells and $\mathrm{B}$ cells (Ishida et al. 1992; Shinohara et al. 1994; Agata et al. 1996; Finger et al. 1997; Vibhakar et al. 1997). When PDCD1 binds to its ligands PDL-1 or PDL-2, PDCD1 inhibits lymphocyte proliferation and cytokine production of previously activated lymphocytes (Bennett et al. 2003). Considering the fact that PDCD1 deficient mice with a C57BL/ 6 background show lupus-like glomerulonephritis and progressive arthritis (Nishimura et al. 1999), it is believed that PDCD1 is a necessary negative regulator to maintain peripheral tolerance and is a key molecule in the development of autoimmune disease.

Prokunina et al. (2002) first described that a regulatory single-nucleotide polymorphism (SNP) in the PDCD1 gene, named PD-1.3 located in intron 4 (rs11568821), was associated with susceptibility to systemic lupus erythematosus (SLE) in Europeans and Mexicans and allele A of SNP PD-1.3 disrupted binding of the Runtrelated transcription factor 1 to the enhancer, and thereby, altered the regulation of gene expression. They also reported that this PD-1.3A was associated with RA in Swedish patients negative for both rheumatoid factor 
(RF) and the shared epitope (Prokunina et al. 2004). To the contrary, the PD-1.3A was significantly less frequent in Spanish SLE patients than in controls (Ferreiros-Vidal et al. 2004). In Asian, allele T of another SNP, PD-1.5C/ $\mathrm{T}$ in exon 5 (rs2227981; alanine $\rightarrow$ alanine), was reported to be associated with the development of RA in Chinese patients living in Taiwan (Lin et al. 2004). A recent study by Kong et al. demonstrated that SNP PD1.3 was nonpolymorphic among both patients with RA and controls in Hong Kong Chinese, and that PD-1.1 G/ $\mathrm{A}$ in the promoter (position -531 from the translation start) was associated with RA patients in their population. They also investigated whether the haplotype containing PD-1.1G and PD-1.5T was a risk factor for RA (Kong et al. 2005).

Although these results indicate that $P D C D 1$ is a candidate gene for RA, the association of $P D C D 1$ with RA remains controversial, probably owing to ethnic differences. Replicating findings both in independent population samples from the same ethnic group and from the other ethnic group is helpful in establishing genetic associations. Therefore, in order to validate the original findings, we undertook a large population-based study on the three reported SNPs including PD-1.1 G/A, PD-1.3 G/A and PD-1.5 $\mathrm{C} / \mathrm{T}$ to investigate whether $P D C D 1$ is associated with RA in a Japanese population.

\section{Materials and methods}

Subjects and disease criteria

This present study is part of a RA cohort project with an enrollment of nearly 5,000 patients established by the Institute of Rheumatology, Tokyo Women's Medical University (IORRA: Institute of Rheumatology RA cohort) (Tanaka et al. 2005). Out of the cohort-registered patients, DNA samples were obtained from 1,504 RA patients $[83 \%$ of the patients were female, $88 \%$ were RF positive, and the mean age of the patients was 59.8 years (range 22-87 years)]. Tokyo Women's Medical University Genome Ethics Committee granted approval of this study, and each individual signed an informed consent form after receiving a verbal explanation of the study. The diagnosis of RA was established using the classification criteria of the American College of Rheumatology (formerly, the American Rheumatism Association) (Arnett et al. 1988). Four hundred and forty-nine population-based control DNA samples were obtained from the Pharma SNP consortium (http://www.jpma.or.jp/psc/index.html). All control subjects were matched for sex, ethnic origin and geographical area. The mean age of the controls was 36.4 years (range $20-74$ years).
Genotyping of PD-1.1, PD-1.3 and PD-1.5

Genotypings were performed using the TaqMan fluorogenic $5^{\prime}$ nuclease assay (Applied Biosystems, Tokyo, Japan). The final volume of polymerase chain reaction (PCR) was $5 \mu$, containing $2 \mathrm{ng}$ of genomic DNA and $2.5 \mu \mathrm{l}$ of TaqMan Universal PCR Master Mix (2X), with $0.125 \mu$ l of $40 X$ Assay Mix or $0.25 \mu \mathrm{l}$ of $20 \mathrm{X}$ Assay Mix. The sequences of the primers and probes were as follows: PD-1.1; forward primer, 5'-TGCCCCAGCGAAGGG-3', reverse primer, 5' CAGGTGCCTGGCCTCTG-3', A-probe, 5'-VIC-CCTTCC TGGCCCATC-NFQ-3' and G-probe, 5'-FAM-CTTCC CGGCCCATC-NFQ-3': PD-1.3; forward primer, 5'-CCTC AATCCCTAAAGCCATGATCTG-3' ${ }^{\prime}$, reverse primer, $5^{\prime}-\mathrm{C}$ AGGCAGGCACACACATG-3', G-probe, 5'-VIC-ACCTG CGGTCTCC-NFQ-3' and A- probe, $5^{\prime}$-FAM-ACCTGCAG TCTCC-NFQ-3': PD-1.5; forward primer, 5'-GCGGAATG GGCACCTCAT-3', reverse primer, 5'-TCCATCCTCAGG CCTCAGT-3', C-probe, 5'-VIC-CGTCGGCTGAGCC-NF Q-3' and T- probe, 5'-FAM-CCGTCAGCTGAGCC-NFQ$3^{\prime}$. Thermal cycle conditions were as follows: $50^{\circ} \mathrm{C}$ for $2 \mathrm{~min}$ and $95^{\circ} \mathrm{C}$ for $10 \mathrm{~min}$, followed by 40 cycles of $92^{\circ} \mathrm{C}$ for $15 \mathrm{~s}$ and $60^{\circ} \mathrm{C}$ for $1 \mathrm{~min}$. All PCRs were performed using 384-well plates by a Dual 384-Well GeneAmp PCR System 9700 (Applied Biosystems), and endpoint fluorescent readings were performed on an ABI PRISM 7900 HT Sequence Detection System (Applied Biosystems).

Statistical analysis

Associations of the SNPs or haplotypes with RA patients were estimated by the chi-square test using the R software package, version 2.4.1 (http://www.r-project.org/). Statistical power was also calculated using the R. Stratification analysis was used to investigate the effect of RF. Haplotypes were constructed using the expectation-maximization algorithm implemented in the PENHAPLO program (Ito et al. 2004). Since the aim of this study was to confirm an association, no Bonferroni correction was applied; statistical significance was set at 5\% level.

\section{Results}

Genotyping

Allele frequencies for PD-1.1 and PD-1.5 were in HardyWeinberg equilibrium in both the patients and the controls. Since PD-1.3 was non-polymorphic among the patients and controls, which was consistent with the result of the Hong Kong Chinese (Kong et al. 2005), PD-1.3 was excluded from further analysis. 
The data summarized in Table 1 show the genotype distributions and allelic frequencies of patients and controls. The allelic frequencies of PD-1.1 and PD-1.5 were compared between patients and controls. We did not observe any significant difference in allelic frequencies of PD-1.1 polymorphism $(P=0.42$, OR 1.06 , 95\% CI $0.91-$ 1.24) or PD-1.5 polymorphism $(P=0.94$, OR $1.01,95 \% \mathrm{CI}$ 0.85-1.19) between RA patients and controls. To assess the possible implication of PD-1.1 and PD-1.5 polymorphisms in RA clinical course, we additionally stratified RA patients according to the presence of the RF. Although a trend for an association was observed between PD-1.1 polymorphism and RA patients negative for RF, it was not statistically significant $(P=0.08$, OR $0.80,95 \%$ CI $0.62-1.03)$.

\section{Haplotype analysis}

Four haplotypes between SNPs PD-1.1 and PD-1.5 were constructed. The SNPs were in strong linkage disequilibrium, with $D^{\prime}$ value 0.93 . No significant difference in the overall frequencies of the haplotypes was observed between RA patients and controls (Table 2).

\section{Discussion}

This study is the first to investigate the involvement of PDCD1 polymorphisms in genetic susceptibility to RA in a Japanese population. Despite its associations with RA in Caucasian and Chinese populations, our results suggest that these SNPs are not associated with the susceptibility to RA in the Japanese population. Haplotype analysis also could not detect the association of $P D C D 1$ with RA in contrast to the previous report (Kong et al. 2005).

Failure to confirm a previously identified association is not unusual in the search for genetic determinants of common diseases. It might arise due to the following reasons. First, ethnic differences may play a role in the conflicting results of association studies. A replication study using a population with a different ethnicity allows us to know whether a reported association is caused by a common variance through different ethnicities. In the case of PDCD1 and RA, PD-1.3, which is the most significantly associated SNP in Swedish RA patients, is not polymorphic in the studied Japanese population. It may be possible that the polymorphisms studied in the Japanese population have

Table 1 Distribution of $P D C D 1$ polymorphisms in RA patients and controls

\begin{tabular}{|c|c|c|c|c|c|c|c|c|}
\hline \multirow[t]{2}{*}{ SNP ID } & & \multicolumn{5}{|c|}{ Genotype of patients } & \multicolumn{2}{|c|}{ Allele 1 versus allele $2^{\mathrm{a}}$} \\
\hline & & $1 / 1$ & $1 / 2$ & $2 / 2$ & Total & MAF & OR $(95 \% \mathrm{CI})$ & $P$ \\
\hline \multirow[t]{4}{*}{ PD-1.1 } & Patients & 360 & 722 & 392 & 1,474 & 0.511 & $1.06(0.91-1.24)$ & 0.42 \\
\hline & $\mathrm{RF}+$ & 307 & 631 & 360 & 1,298 & 0.520 & $1.11(0.95-1.29)$ & 0.20 \\
\hline & $\mathrm{RF}-$ & 53 & 91 & 32 & 176 & 0.440 & $0.80(0.62-1.03)$ & 0.08 \\
\hline & Controls & 124 & 203 & 120 & 447 & 0.496 & & \\
\hline \multirow[t]{4}{*}{ PD-1.5 } & Patients & 714 & 615 & 156 & 1,485 & 0.312 & $1.01(0.85-1.19)$ & 0.94 \\
\hline & $\mathrm{RF}+$ & 631 & 537 & 142 & 1,310 & 0.313 & $1.01(0.86-1.20)$ & 0.89 \\
\hline & $\mathrm{RF}-$ & 83 & 78 & 14 & 175 & 0.303 & $0.96(0.73-1.27)$ & 0.79 \\
\hline & Controls & 217 & 178 & 49 & 444 & 0.311 & & \\
\hline
\end{tabular}

The major allele in controls was always referred to as allele 1 and the minor allele as allele 2. SNP single-nucleotide polymorphism; $M A F$ minor allele frequency; OR odds ratio; 95\% CI 95\% confidence interval; $R F$ rheumatoid factor

${ }^{a}$ Distribution of the frequency of allele 1 versus allele 2 in the cases compared with the controls

Table 2 PDCD1 haplotype structure and frequencies

\begin{tabular}{|c|c|c|c|c|c|c|}
\hline \multirow[t]{2}{*}{ Haplotype } & \multicolumn{2}{|l|}{ SNP ID } & \multicolumn{4}{|c|}{ Haplotype comparison $^{\mathrm{a}}$} \\
\hline & PD-1.1 & PD-1.5 & Patients & Controls & OR $(95 \% \mathrm{CI})$ & $P$ \\
\hline 1 & A & $\mathrm{C}$ & 0.481 & 0.492 & $0.95(0.82-1.11)$ & 0.57 \\
\hline 2 & A & $\mathrm{T}$ & 0.009 & 0.012 & $0.81(0.39-1.80)$ & 0.57 \\
\hline 3 & G & $\mathrm{C}$ & 0.207 & 0.196 & $1.06(0.88-1.29)$ & 0.54 \\
\hline 4 & $\mathrm{G}$ & $\mathrm{T}$ & 0.302 & 0.299 & $1.02(0.86-1.20)$ & 0.87 \\
\hline
\end{tabular}

SNP single-nucleotide polymorphism; OR odds ratio; 95\% CI 95\% confidence interval

${ }^{a}$ Each haplotype was compared with the other haplotypes combined 
no primary effect on susceptibility since the etiological mutation present in the Swedish is not present in the population. However, the studies by Lin et al. and Kong et al. identified the association between RA and PDCDI in East Asian populations. Since subjects in the present study had similar ethnic backgrounds as those in the Asian studies, and the polymorphisms studied in those East Asian studies were covered in this study, it might be possible that population-specific differences in East Asians caused this discrepancy.

Second, it could be possible that our results might have arisen due to a lack of sufficient statistical power. It is well known that the HLA locus, which has been estimated to account for one-third of the genetic component, has a great impact on RA susceptibility, and that each of the disease-associated genes outside the HLA region may have a limited relative risk for the disease. For this reason, identifying susceptible genes outside the HLA region in an association study needs a large sample size to obtain an adequate statistical power. Our study used a large sample size (1,504 cases and 449 controls) that was much larger than previous East Asian studies by Lin et al. (84 cases and 135 controls) (Lin et al. 2004) and Kong et al. (180 cases and 647 controls) (Kong et al. 2005), and our study was designed to have sufficient power to detect the effect of PD-1.1 polymorphism conferring an OR of $0.63(P<0.05$, with a $49 \%$ frequency of the allele in controls) or PD-1.5 polymorphism conferring an OR of $1.94(P<0.05$, with a $31 \%$ frequency of the allele in controls).

In conclusion, using a large set of Japanese patients and population controls, we could not confirm associations of PD-1.1, -1.3 and -1.5 polymorphisms in PDCDI with RA patients at least in the Japanese population. However, further studies are required to test the possibility that other polymorphisms within the PDCDl gene may be involved in the susceptibility to RA.

Acknowledgments We thank all DNA donors for making this study possible. We appreciate Dr. Iikuni for her assistance in preparing the manuscript and Ms. Yamanaka for her technical efforts. This work was supported by a grant provided by the Japan Orthopaedics and Traumatology Foundation (to K.I.) and a Grant-in-Aid for Young Scientists from the Japanese Ministry of Education, Culuture, Sports, Science and Technology (to K.I.). We are also grateful to 36 pharmaceutical companies for the support of the IORRA study, and Prof. Taniguchi and other members of our Institute for their efforts on the IORRA cohort.

\section{References}

Agata Y, Kawasaki A, Nishimura H, Ishida Y, Tsubata T, Yagita H, Honjo T (1996) Expression of the PD-1 antigen on the surface of stimulated mouse T and B lymphocytes. Int Immunol 8:765-772
Arnett FC, Edworthy SM, Bloch DA, McShane DJ, Fries JF, Cooper NS, Healey LA, Kaplan SR, Liang MH, Luthra HS, et al (1988) The American Rheumatism Association 1987 revised criteria for the classification of rheumatoid arthritis. Arthritis Rheum 31:315-324

Bennett F, Luxenberg D, Ling V, Wang IM, Marquette K, Lowe D, Khan N, Veldman G, Jacobs KA, Valge-Archer VE, Collins M, Carreno BM (2003) Program death-1 engagement upon TCR activation has distinct effects on costimulation and cytokinedriven proliferation: attenuation of ICOS, IL-4, and IL-21, but not CD28, IL-7, and IL-15 responses. J Immunol 170:711-718

Ferreiros-Vidal I, Gomez-Reino JJ, Barros F, Carracedo A, Carreira P, Gonzalez-Escribano F, Liz M, Martin J, Ordi J, Vicario JL, Gonzalez A (2004) Association of PDCD1 with susceptibility to systemic lupus erythematosus: evidence of population-specific effects. Arthritis Rheum 50:2590-2597

Finger LR, Pu J, Wasserman R, Vibhakar R, Louie E, Hardy RR, Burrows PD, Billips LG (1997) The human PD-1 gene: complete cDNA, genomic organization, and developmentally regulated expression in B cell progenitors. Gene 197:177-187

Ishida Y, Agata Y, Shibahara K, Honjo T (1992) Induced expression of PD-1, a novel member of the immunoglobulin gene superfamily, upon programmed cell death. Embo J 11:3887-3895

Ito T, Inoue E, Kamatani N (2004) Association test algorithm between a qualitative phenotype and a haplotype or haplotype set using simultaneous estimation of haplotype frequencies, diplotype configurations and diplotype-based penetrances. Genetics 168:2339-2348

Kong EK, Prokunina-Olsson L, Wong WH, Lau CS, Chan TM, Alarcon-Riquelme M, Lau YL (2005) A new haplotype of PDCD1 is associated with rheumatoid arthritis in Hong Kong Chinese. Arthritis Rheum 52:1058-1062

Lin SC, Yen JH, Tsai JJ, Tsai WC, Ou TT, Liu HW, Chen CJ (2004) Association of a programmed death 1 gene polymorphism with the development of rheumatoid arthritis, but not systemic lupus erythematosus. Arthritis Rheum 50:770-775

Nishimura H, Nose M, Hiai H, Minato N, Honjo T (1999) Development of lupus-like autoimmune diseases by disruption of the PD-1 gene encoding an ITIM motif-carrying immunoreceptor. Immunity 11:141-151

Prokunina L, Castillejo-Lopez C, Oberg F, Gunnarsson I, Berg L, Magnusson V, Brookes AJ, Tentler D, Kristjansdottir H, Grondal G, Bolstad AI, Svenungsson E, Lundberg I, Sturfelt G, Jonssen A, Truedsson L, Lima G, Alcocer-Varela J, Jonsson R, Gyllensten UB, Harley JB, Alarcon-Segovia D, Steinsson K, Alarcon-Riquelme ME (2002) A regulatory polymorphism in PDCD1 is associated with susceptibility to systemic lupus erythematosus in humans. Nat Genet 32:666-669

Prokunina L, Padyukov L, Bennet A, de Faire U, Wiman B, Prince J, Alfredsson L, Klareskog L, Alarcon-Riquelme M (2004) Association of the PD-1.3A allele of the PDCD1 gene in patients with rheumatoid arthritis negative for rheumatoid factor and the shared epitope. Arthritis Rheum 50:1770-1773

Shinohara T, Taniwaki M, Ishida Y, Kawaichi M, Honjo T (1994) Structure and chromosomal localization of the human PD-1 gene (PDCD1). Genomics 23:704-706

Tanaka E, Saito A, Kamitsuji S, Yamada T, Nakajima A, Taniguchi A, Hara M, Tomatsu T, Yamanaka H, Kamatani N (2005) Impact of shoulder, elbow, and knee joint involvement on assessment of rheumatoid arthritis using the American College of Rheumatology Core Data Set. Arthritis Rheum 53:864-871

Vibhakar R, Juan G, Traganos F, Darzynkiewicz Z, Finger LR (1997) Activation-induced expression of human programmed death-1 gene in T-lymphocytes. Exp Cell Res 232:25-28 